\title{
Real-Time Three-Dimensional Echocardiographic Evaluation of Global and Regional Left Ventricular Function
}

\author{
C Corsi ${ }^{1,2}$, RM Lang ${ }^{1}$, F Veronesi $^{2,3}$, L Weinert $^{1}$, EG Caiani ${ }^{3}$, \\ $\mathrm{P}_{\text {MacEneaney }}{ }^{1}, \mathrm{C}_{\text {Lamberti }}{ }^{2}, \mathrm{~V}$ Mor-Avi ${ }^{1}$ \\ ${ }^{1}$ Noninvasive Cardiac Imaging Laboratories, University of Chicago, IL, USA \\ ${ }^{2}$ Department of Electronics, Computer Science and Systems, University of Bologna, Italy \\ ${ }^{3}$ Biomedical Engineering Department, Polytechnic of Milan, Italy
}

\begin{abstract}
Quantification of left ventricular ( $L V)$ function from the analysis of real-time $3 D$ echocardiographic (RT3DE) data does not fully exploit the dynamic volumetric information contained in these data. Accordingly, we developed a volumetric analysis technique aimed at quantification of global and regional LV function. RT3DE images were analyzed using custom software to extract global and partial volumes and regional wall motion (RWM) throughout the cardiac cycle. RT3DE were compared point-by-point with magnetic resonance (MR) data (linear regression and Bland-Altman analysis) and the feasibility of automated detection of RWM abnormalities was tested by comparison with expert reading of $2 D$ echocardiographic images. $L V$ volumes correlated highly with MR with minimal bias and narrow limits of agreement. RWM correlated highly in basal and mid segments. The automated detection of RWM agreed with expert interpretation in $86 \%$ of $L V$ segments. Volumetric analysis of RT3DE data is clinically feasible and allows fast and accurate assessment of cardiac function and automated detection of RWM abnormalities.
\end{abstract}

\section{Introduction}

Conventional visual interpretation of two-dimensional echocardiographic (2DE) images aimed at the evaluation of left ventricular (LV) function uses only partial information contained in specific cross-sectional planes and relies on the reader's experience and ability to effectively integrate spatial and temporal information. Three-dimensional (3D) imaging based on off-line reconstruction from multiple planes overcomes some of these limitations, since it provides more complete information about cardiac anatomy and function. Nevertheless, the practicality of this methodology for clinical use has been hindered by the cumbersome and lengthy data acquisition coupled with the time-consuming analysis.
The recently developed real-time three-dimensional echocardiographic (RT3DE) imaging technology offers significant advantages over both the $2 \mathrm{D}$ and $3 \mathrm{D}$ imaging in this context. Several recent studies have demonstrated the potential improvements in the evaluation of global [13] and regional [4] LV function form RT3DE data. However, analysis procedures used in these studies did not fully exploit the volumetric information contained in the RT3DE datasets and therefore were fraught with some of the same limitations as the techniques used to analyze $2 \mathrm{D}$ and $3 \mathrm{D}$ images.

We hypothesized that our recently developed technique for direct 3D detection of the endocardial surface [5] could be applied to RT3DE datasets throughout the cardiac cycle and thus lead to an alternative approach for fast, easy and objective dynamic quantification of LV volume (LVV) and also provide the basis for objective assessment of regional wall motion (RWM). Accordingly, our goal was to test the clinical feasibility of this technique and validate it against cardiac magnetic resonance (MR) imaging.

\section{Methods}

The study design included 3 protocols, which were carried out in 30 subjects $(19 \hat{\circ}, 11$; ; age: $58 \pm 19 \mathrm{yrs})$.

MR data were obtained using a 1.5 Tesla scanner (GE). Six to ten short-axis slices were acquired during 12 -second breath-holds, from the atrioventricular ring to the apex ( $9 \mathrm{~mm}$ slice thickness, no gaps) with a temporal resolution of 20 frames per cardiac cycle. Images were analyzed using commercial software (MASS Analysis, Medis). In every slice, LV endocardial contours were traced semi-automatically frame-by-frame, with the papillary muscles included in the LV cavity, and manually corrected when necessary, resulting in LV cross-sectional area over time for each slice. Global LVV was computed using a disk-area summation method, resulting in volume time curves. In addition, standard segmentation procedure was used to obtain RWM over time (in $\mathrm{mm}$, relative to end-diastolic (ED) endocardial 
boundary position) for anteroseptal, anterior, lateral posterior, inferior and septal segments in each slice. To obtain MR reference for partial volume at 3 different levels of the LV and RWM time curves, the number of slices covering the LV, which differed from patient to patient, was divided by 3 . The resultant number was used to create weight coefficients that reflected the contribution of each slice toward the calculation of partial volumes and RWM.

Transthoracic harmonic RT3DE datasets were acquired using the SONOS 7500 system (Philips) equipped with a fully sampled matrix array transducer (X4). In addition, 2D images were acquired in apical 2-, 3- and 4-chamber views. The RT3DE datasets were analyzed using custom software for endocardial surface detection, based on the level set approach. Surface detection was performed with the papillary muscles included in the LV cavity to be consistent with MR. This procedure was applied to every consecutive frame, resulting in global LVV time curves. Subsequently, the LV endocardial surface was automatically segmented throughout the cardiac cycle (figure 1), while applying

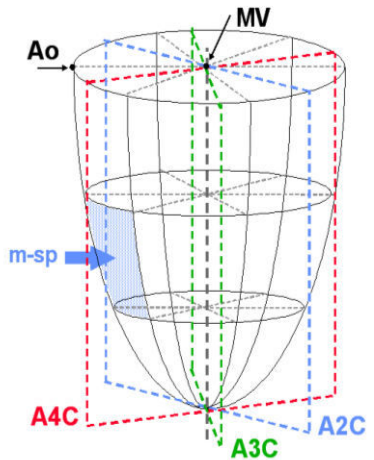

Figure 1. Schematic display of the $3 \mathrm{D}$ segmentation model. optical flow techniques $[6,7]$ to follow the center of the mitral annulus, the tip of the apex and the center of the aortic annulus. Partial volumes inside the endocardial surface were calculated for basal, mid and apical levels for each consecutive frame, resulting in partial volume time curves. Moreover, regional volume was calculated throughout the cardiac cycle and used to calculate for each segment the time interval between the ECG Rwave and the time when the smallest regional LV volume was reached. The standard deviation of these time intervals calculated for the 18 segments was used as an index of LV systolic asynchrony (ISA) in each patient. In addition, for each radial segment and for all consecutive frames, the distance between the long axis and each surface voxel was computed and averaged for all surface voxels, resulting in the regional radial dimension (RRD). RWM was calculated as the difference between ED and current RRD, resulting in 18 RWM time curves. In addition, peak RWM (difference between ED and endsystolic (ES) wall motion) was divided by RRD at ED to obtain regional shortening fraction (RSF). To allow pointby-point comparisons between subjects and between techniques (RT3DE vs. MR), all the RT3DE-derived time curves were resampled at 20 frames per cardiac cycle.

Protocol 1 was designed to test and validate RT3DEbased continuous measurements of global LV volume, partial volumes, and RWM time-curves against MR reference values. Sixteen patients $(10 \hat{\jmath}, 6 \circ$; age: $52 \pm 17$ yrs) were included in the protocol. RT3DE data acquisition was performed on the same day as the MR study. For volume measurements, the comparisons included linear regression and Bland-Altman analyses. Percent discordance between the two techniques was calculated as the point-by-point sum of absolute differences between RT3DE and MR values, normalized by the point-by-point sum of MR values. For wall motion curves, comparisons were performed for peak systolic values only. In addition, segments at each LV level were grouped together and analyzed separately.

In protocol 2 we tested the ability of our technique to discern populations with known differences in LV performance by comparing global and regional indices of systolic and diastolic function obtained in a group of 9 patients with dilated cardiomyopathy (DCM) $(6 \hat{0}, 3 \circ$; age: $58 \pm 14$ yrs) with those obtained in a group of 9 agematched subjects with normal global LV function $(5 \hat{\jmath}$, 4; age: $51 \pm 17$ yrs). For each subject, global ES and ED volumes (ESV, EDV) were extracted from the global LVV curves and used to calculate the ejection fraction (EF). Peak ejection rate (PER) and peak rapid and atrial filling rates (PRFR, PAFR) were obtained from the time derivative of the global LVV curve and normalized by EDV. In addition, ISA and RSF were computed for each subject. All indices were compared between the patients with DCM and their age-matched normal counterparts using one-tailed paired t-test.

Protocol 3 was designed to test the feasibility of automated detection of RWM abnormalities using quantitative analysis of RT3DE data. The protocol included 18 patients: 11 patients with coronary artery diseases (CAD) $(6 \hat{\jmath}, 5+$; age: $70 \pm 16$ yrs) and 7 healty subjects $(4 \hat{\circ}, 3 \circ$; age: $40 \pm 16$ yrs), who were used as a normal control group. Apical 2-, 3- and 4-chamber 2D images in the 11 patients were reviewed by an experienced echocardiographer who graded RWM in each segment as normal or abnormal. The mean RSF-1SD obtained from RT3DE data in the normal control group was used as a threshold for automated classification of each segment as normal or abnormal. The percent of segments where automated classification agreed with the interpretation of 2D images was computed, as well as percent of false positive and false negative interpretations.

\section{Results}

RT3DE imaging was feasible in all study subjects. The average time required to analyze a single time frame, including data retrieval, frame selection, surface detection 

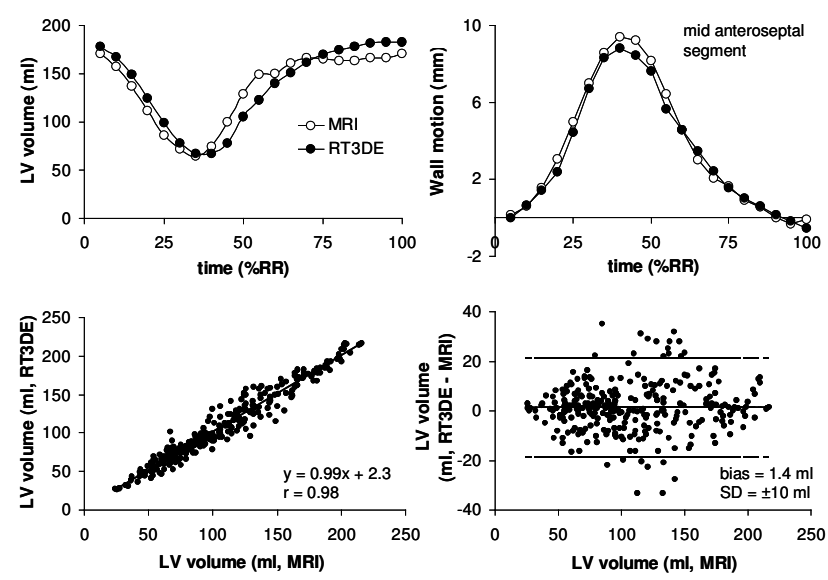

Figure 2. Top panels: Example of time curves obtained in one patient from RT3DE and MR data; Bottom panels: Results of linear regression and Bland Altman analyses of point-by-point measurements of global LV volume.

and segmentation, computation of volume and wall motion, was approximately 5 minutes on a personal computer (Pentium II, 755MHz), of which only $30 \mathrm{sec}$ were required for the computations.

In protocol 1, all curves showed good agreement between RT3DE and MR (figure 2, top panels). For global LVV, the high level of agreement was confirmed by the statistical analysis in the entire study group (figure 2 , bottom panels). The percent discordance was only $6 \pm 3 \%$. For the partial volumes, the correlation between RT3DE and MR ranged between good and excellent, but showed differences between the basal $(r=0.96)$, mid $(\mathrm{r}=0.97)$ and apical $(\mathrm{r}=0.81)$ levels. Bland Altman analysis showed only small biases of $-0.5,1.7$ and $1.1 \mathrm{ml}$ for the basal, mid and apical levels, respectively $(-1 \%, 4 \%$ and $6 \%$ of the corresponding mean MR partial volume), and narrow limits of agreement at 11,8 and $10 \mathrm{ml}$. The calculated percent discordance values were $9 \pm 6 \%, 8 \pm 4 \%$ and $21 \pm 12 \%$ respectively. The correlation between MR and RT3DE-derived peak systolic RWM values was modest $(r=0.69)$ when calculated for all LV segments combined, with a bias of $-1.3 \mathrm{~mm}(-15 \%$ of the mean MR value) and $95 \%$ limits of agreement at $\pm 4.6 \mathrm{~mm}$. With the apical segments excluded, the agreement with MR was markedly improved $(r=0.89)$ with only a minimal bias of $-0.5 \mathrm{~mm}$ (only $-6 \%$ of the mean MR value) and $95 \%$ limits of agreement at $\pm 2.6 \mathrm{~mm}$. The percent discordance was $22 \pm 25 \%$ for all segments combined and only $13 \pm 12 \%$ for the basal and mid LV segments.

In protocol 2, the group composite LVV time curves demonstrated the inter-group differences with no overlap at any phase of the cardiac cycle, and the standard deviation (bigger in the DCM group) reflected the variable severity of the disease in these patients. The results of statistical analysis showed that all parameters of

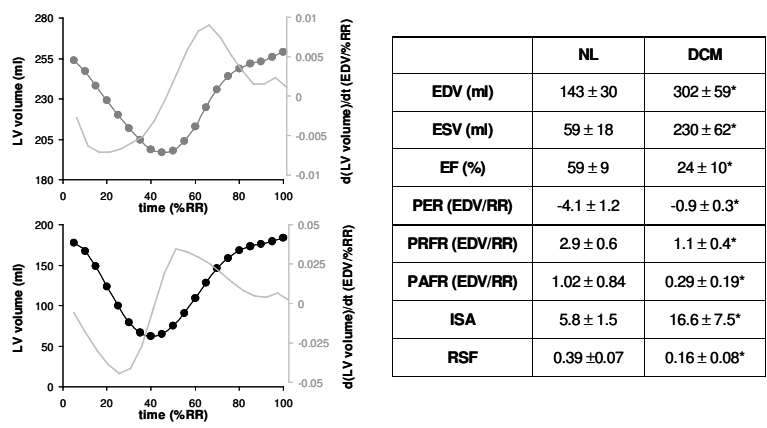

Figure 3. Left: Example of global LVV time curves obtained in a patient with DCM (top) and an age-matched normal subject (bottom) shown with the time-derivatives (solid lines without symbols, secondary y-axes). Right: Results of statistical analysis on all parameters of global and regional LV systolic and diastolic function $\left({ }^{*} \mathrm{p}<0.05\right)$.
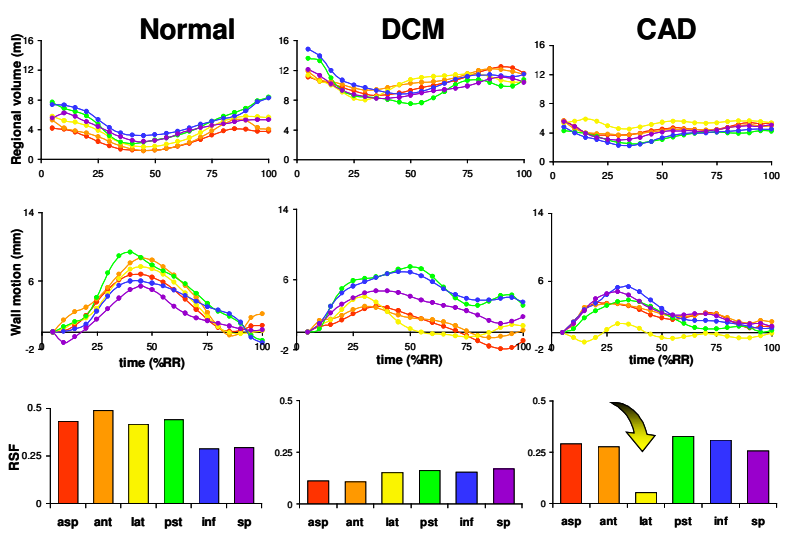

Figure 4. Examples of regional volume (top), RWM (center) and RSF (bottom) in the apical segments, obtained in a normal subject (left panels), a DCM patient (middle panels) and a CAD patient (right panels) with a RWM abnormality in the lateral wall (arrow).

global and regional LV systolic and diastolic function calculated from volume and wall motion time curves were significantly different between the two groups (figure 3). Figure 4 shows an example of regional volume and RWM time curves and RSF obtained in a normal subject (left) and a patient with DCM (center). As expected, regional volumes in the patient with DCM were markedly increased compared to the normal subject. Also, both regional volume and RWM curves showed less synchronous pattern of contraction in the patient with DCM than in the normal subject. Despite the morphological similarity between the RWM curves in these two subjects, RSF was smaller in all segments, demonstrating on a regional basis the reduced $\mathrm{LV}$ function in patients with DCM.

For protocol 3, figure 4 (right) shows data obtained in a patient with CAD and a RWM abnormality in the apical-lateral segment, as reflected by the low amplitude 
and asynchronous motion noted in both regional volume and RWM time curves and by the markedly reduced RSF value in this segment. RFS values obtained in the normal control group allowed correct automated classification of $170 / 198$ (86\%) segments as normal or abnormal in agreement with the expert wall motion scores based on visual interpretation of 2D images. Eighteen of 198 segments $(9.1 \%)$ were misclassified as abnormal and only $10 / 198$ segments $(5.1 \%)$ were falsely graded as normal.

\section{Discussion and conclusions}

This study aimed at direct detection of the endocardial surface in the 3D domain to allow the volumetric information contained in the RT3DE dataset to be fully exploited, without either the need for plane selection or geometric modeling. In this study, we developed, validated and tested in the clinical setting a new technique for fast quantification of global and regional LV function from RT3DE data. We tested our hypotheses in three separate protocols. In protocol 1, we validated against MR reference the three variables that could be compared to data provided by the commercial MR analysis package. Direct point-by-point comparisons of global LVV and RWM measurements showed high levels of agreement with MR and proved the ability of our technique to access information about regional LV function as readily as the current standard reference technique. The relatively low correlation and high percent discordance for the apical partial volumes and RWM can be explained by two factors: (i) near the apex, endocardial boundary is less well defined than at higher levels of the left ventricle for both RT3DE and MR, which is likely to affect the accuracy of endocardial detection by both techniques; (ii) the use of fixed number of slices of fixed thickness by MR disregards systolic longitudinal shortening which is likely to affect the accuracy of the MR measurements. In this regard, MR imaging cannot provide perfectly accurate reference even if its use as such is common and well established. Protocol 2 was designed as a first step for clinical testing of our technique by quantifying regional LV RWM and its asynchrony from clinical RT3DE data. The availability of regional volume curves gave us access to regional timing rather than just magnitude information, which was used to successfully diagnose LV systolic asynchrony known to be prevalent in DCM patients [8]. Albeit in a small group of patients, the ability to detect RWM abnormalities was further proved in protocol 3, which was aimed at establishing the clinical usefulness of segmental analysis of RT3DE data in CAD patients. Importantly, this protocol constitutes the first attempt to objectively interpret LV RWM from RT3DE data using a technique that is fully automated once the endocardial segmentation is complete.

In conclusion, we found that volumetric analysis of
RT3DE data for quantitative assessment of LV function without the limitations of 2D imaging or 2D-based analysis of $3 \mathrm{D}$ data is feasible. This approach allows the quantification of global and regional LV function, including the magnitude and timing of RWM. This analysis holds promise for multiple clinical applications in a variety of disease states affecting the myocardium. Witnessing how the RT3DE technology is gaining widespread use and anticipating the development of commercial software for true volumetric analysis, we believe that this combination will become routine in the clinical practice.

\section{References}

[1] Sugeng L, Weinert L, Lang RM. Left ventricular assessment using real-time three-dimensional echocardiography. Heart 2003; 89 Suppl 3:iii29-iii36.

[2] Mor-Avi V, Sugeng L, Weinert L, MacEneaney P, Caiani EG, Kock R, Salgo IS, Lang RM. Fast measurement of left ventricular mass with real-time three-dimensional echocardiography: comparison with magnetic resonance imaging. Circulation 2004; 110:1814-8.

[3] Kuhl HP, Schreckenberg M, Rulands D, Katoh M, Schafer W, Schummers G, Bucker A, Hanrath P, Franke A. Highresolution transthoracic real-time three-dimensional echocardiography: quantitation of cardiac volumes and function using semi-automatic border detection and comparison with cardiac magnetic resonance imaging. J Am Coll Cardiol 2004; 43:2083-90.

[4] Collins M, Hsieh A, Ohazama CJ, Ota T, Stetten G, Donovan CL, Kisslo J, Ryan T. Assessment of regional wall motion abnormalities with real-time 3-dimensional echocardiography. J Am Soc Echocardiogr 1999; 12:7-14.

[5] Corsi C, Saracino G, Sarti A, Lamberti C. Left ventricular volume estimation for real-time three-dimensional echocardiography. IEEE Trans Med Imaging 2002; 21:1202-8.

[6] Barron JL, Fleet DJ, Beauchemin SS. Performance of optical flow techniques. International Journal of Computer Vision 77, 12-43. 1994.

[7] Lucas BD, Kanade T. An iterative image registration technique with an application to stereo vision. DARPA Image Understanding Workshop, 121-130. 1981.

[8] Godoy IE, Mor-Avi V, Weinert L, Vignon P, Koncarz C, Spencer KT, Lang RM. Use of color kinesis for evaluation of left ventricular filling in patients with dilated cardiomyopathy and mitral regurgitation. J Am Coll Cardiol 1998; 31:1598-606.

Address for correspondence

Victor Mor-Avi

University of Chicago Medical Center, MC5084, 5841 S. Maryland Ave., Chicago, Illinois 60637, USA vmoravi@medicine.bsd.uchicago.edu 